\title{
Un espace potentiel au collège
}

\author{
Les espaces et les temps informels, \\ lieux et moments adolescents
}

Aurélie Maurin

«Pour être soutenable sans désastre, ce "moment fondateur adolescent" doit s'inscrire dans des dispositif partagés, anthropologiques et culturels, variables d'une société à l'autre. » (Masson, 2004.)

\section{MOMENT ÉCOLE, MOMENT ADOLESCENT : CONSTRUCTION D'UN VIVRE ENSEMBLE}

Le contexte qui sera le nôtre est celui de ce «moment adolescent », tel que le définit Antoine Masson c'est-à-dire « une ponctualité temporelle, une puissance de mouvement et une phase dans le développement » qui serait « paradigmatique de tout moment de fondation subjective où l'être sujet se trouve à travers ce qui le saisit » (Masson, 2004, pp. 3-4). Dans la France d'aujourd'hui, pour la quasi-totalité des jeunes gens, ce moment de l'adolescence prend place, au moins jusqu'à 16 ans et pour une large part, dans ce que ce numéro tente de circonscrire sous la formule parallèle de «moment école».

L'école, terme générique comprenant l'ensemble des institutions scolaires (école primaire, collège, lycée...), pourrait en effet répondre, au moins pour partie, d'un « espace collectif où le sujet est défini par plusieurs occurrences de l'Autre » (Dufour, 2003, p. 48). C'est en cela qu'elle nous semble pouvoir symboliquement accueillir le « moment adolescent » dans sa complexité. Mais avant cela, l'école doit être perçue et décrite pour ce qu'elle est, le plus simplement : un lieu de vie pour près de douze millions d'élèves, âgés en moyenne de 3 à 21 ans, auxquels il faut adjoindre plus d'un million d'adultes professionnels. C'est donc un cinquième de la population française, sans compter les parents, qui passent plusieurs heures par jour dans l'antre des institutions scolaires. 
Un espace potentiel au collège

Les adolescents qui seront évoqués et cités plus loin vivent en France, sont âgés de 13 à 16 ans, et passent plus d'un tiers de leur temps à l'intérieur des établissements scolaires où nous les avons rencontrés et où ils sont accueillis de 8 heures le matin jusqu'à 17 heures, et parfois plus tard encore. Certains déjeunent sur place et y restent donc plus de huit heures par jour. Pendant ce temps, ils y étudient, y tissent des liens d'amitié, s'y découvrent des centres d'intérêts, s'y confrontent à l'autre, adulte ou pair, de même sexe ou non, y rencontrent la différence, l'attirance et le rejet, y surprennent le mouvement des corps, s'y construisent dans l'intersubjectivité. En un mot ils y vivent, mais y habitent-ils ? Ce sont plus particulièrement les espaces et les temps que je nomme informels, c'està-dire les couloirs, les halls, les parvis, la récréation, les interclasses (temps de pause, de repas), etc., qui sont susceptibles de faire office d'habitat, et peut-être d'habitacle. C'est en tout cas la thèse qui sera défendue à partir d'éléments d'analyse soutenus par un corpus clinique.

\section{VIVRE ET HABITER DANS UN COLLÈGE SANS ESPACE INFORMEL ?}

Dans le cadre de la recherche présentée ici et qui porte sur les espaces et les temps informels dans les institutions éducatives pour adolescents, j'ai été amenée à imaginer plusieurs dispositifs méthodologiques. Tous furent articulés autour de rencontres avec les adolescents, parfois dans les lieux mêmes qu'ils décrivent comme lieux de vie, où ils se retrouvent, bavardent, flirtent, échangent... Ces rencontres, après un premier temps d'observation, ont pris la forme d'entretiens, de groupes de parole, puis de groupes à médiation autour des plans du collège, avant de m'emmener un peu plus loin et de mettre en place des ateliers de photographie.

J'étayerai essentiellement les analyses qui vont suivre sur un dispositif original de recherche intitulé « collège idéal » qui a réuni plusieurs adolescents autour des plans de réhabilitation de leur collège, afin qu'ils en deviennent les commentateurs-analystes. Ce qui motive ce choix, c'est que ces ateliers ont été menés dans un établissement dont l'architecture peut aisément être qualifiée de précaire en ce qu'elle relève d'un «provisoire qui dure ». Aussi, ce que nous avons pu observer des espaces et des temps informels dans d'autres établissements « classiques », au moins sur ce point, était tout à fait différent dans ce lieu. C'est donc à partir du manque - dont naît le désir, disent les philosophes - que certains éléments d'analyse ont pu être mis au jour.

L'organisation spatiale en enfilade de préfabriqués de plain-pied, comme jetés de part et d'autre d'une allée centrale et débouchant sur un espace plus vaste encadré de grillages donnant sur un parc avoisinant, ne favorise pas l'informalité des entre-deux habituellement reconnus dans les 
points de passages que sont les seuils, les couloirs et les cages d'escaliers. C'est l'absence de cet informel spatial, de ces interstices, ou du moins la non-immédiateté de leur accessibilité physique et psychique, qui me permettra de questionner le rôle fondamental de ceux-ci au cœur des institutions scolaires.

Pour plus de clarté, il me faut expliciter quelques éléments relatifs à la mise en place générale de cet atelier. Tout comme un autre travail mené en parallèle autour de la photographie (Maurin, à paraître en 2010), il repose sur le principe de la projection : ce que l'on dit de ce que l'on perçoit au-dehors parle aussi de ce que l'on ressent et de ce que l'on vit au-dedans. Ce postulat est celui des méthodes cliniques projectives soutenues actuellement par des chercheurs tels que Catherine Chabert (1998), mais aussi celui des méthodes dites «à médiation » qui sont « avant tout un espace d'expérience et un processus transformationnel » devant permettre d'aller « de l'image au symbole», ainsi que les décrit Claudine Vacheret (2002, pp. XII et 4). J'ai repris à mon compte un certain nombre de ces concepts pour imaginer ces deux ateliers. Les plans du « futur collège » et les photographies des lieux de vie pour les adolescents ont été l'occasion de projections mais surtout des prétextes ou plutôt des préalables aux discours, aux récits et aux dialogues.

L'idée de cet atelier a émergé après deux années de recherche dans ce même établissement ${ }^{1}$. Nous, c'est-à-dire moi-même accompagnée d'une coanimatrice ${ }^{2}$, avons décidé de solliciter une classe autour du thème du « collège idéal ». Après une première rencontre en classe entière, nous avons composé des groupes de quatre ou cinq adolescents que nous avons retrouvés les semaines suivantes, installés dans une pièce où était affiché le plan - un original - du futur collège dont la reconstruction allait bientôt commencer, et qui était attendu depuis près de cinq ans.

La question de départ était: «Parlez-nous de votre collège! » La réponse ne tarda pas à se faire entendre et contre toute attente elle ne nous surprit pas : «Il n'y a pas de collège ! », s'exclamèrent-ils. Ce cri, d'alarme mais aussi du cœur, nous y avions déjà été confrontées, les années précédentes dans ce même établissement à l'occasion de groupes de parole dont les thèmes étaient libres. C'est après que les adolescents d'alors nous eurent tenu ce discours, entre souffrance, honte et cynisme, que nous prîmes l'initiative de leur donner la parole plus avant sur ce thème.

1. «Copsy-enfant », La construction de l'identité aujourd'hui. Construction psychique et psychopathologie de l'enfant dans les nouveaux liens familiaux et sociaux, direction scientifique Serge Lesourd, Appel blanc 2005-2008, Agence nationale de la recherche (ANR), voir Gavarini, 2009.

2. Je remercie ici Viviani Do Carmo Hureta. 


\section{Dossier}

Un espace potentiel au collège

Leur collège, détruit quelques années auparavant et depuis lors constitué de préfabriqués nommés «boîtes », « caravanes » ou « cabanes » par les élèves, est défini par eux comme inexistant ou comparé à des détritus, de la matière en décomposition «pourrie », ou décomposable « en carton ». C'est le caractère fragile et délité de celui-ci qui retient particulièrement l'attention. Ainsi parlent-ils de «morceau de collège », de «fragment », et narrent comment les parois leur paraissent poreuses et les exposent à tous les dangers. Pourtant c'est bien la sensation angoissante d'enfermement qui prévaut et laisse émerger quelques comparaisons fortes avec les «camps de concentration », la «prison », les «ghettos »... Autrement dit, il s'agit d'un lieu vécu comme ne pouvant ni contenir, ni protéger, tout en privant de liberté. C'est l'image d'une peau retournée qui vient à l'esprit, son paradoxe serait d'avoir sa face à vif au dehors, exposante, tandis que sa face imperméable serait au-dedans, étouffante.

C'est l'absence de cet informel spatial, de ces interstices, ou du moins la non-immédiateté de leur accessibilité physique et psychique, qui me permettra de questionner le rôle fondamental de ceux-ci au cœur des institutions scolaires.
Le collège à venir est quant à lui d'abord rejeté dans un futur ne les concernant pas: «Quel futur? »; «On sera même pas là »; « Nous on sera au lycée », protestent-ils. Ils relatent alors de concert avoir vu les promesses des adultes s'évanouir d'année en année : «En sixième, on nous a dit : "Quand vous serez en troisième il y aura le nouveau collège", et là on est déjà en quatrième. »Certains affirment même que leurs enfants ne verront pas ce collège « futuriste ». Ce vocable signifiant semble être utilisé à la fois dans le sens de «moderne », ainsi que le laissent présager les plans, mais aussi dans le sens de «non réaliste », en ce qu'il leur apparaît d'une part comme temporellement et spatialement abstrait et donc psychiquement voué au seul imaginaire, et d'autre part comme leur étant non destiné. Outre les espoirs déçus, c'est plus encore dans l'assurance d'y trouver leur place que subsiste le doute. Pour exemple, le corps de bâtiment ayant éveillé le plus la curiosité et généré le plus de discussions est sans conteste l'internat. Celui-ci est destiné à accueillir des élèves d'autres départements qui seront scolarisés dans une section à part, fortement valorisée et valorisante, dite de sports-études. En outre, ce qui a été énoncé par tous les groupes, y compris les adolescents rencontrés les années précédentes, c'est l'absence d'étages dans le présent collège et le vif souhait, voire le besoin, qu'il y en ait dans le futur collège ainsi que dans le lycée qui devra les accueillir dans les années à venir. C'est avec Gaston Bachelard (1957, pp. 24 et 45) et sa «topo-analyse » qu'il est possible de comprendre ces explicitations comme relevant d'une représentation de la «maison-collège » prise dans sa verticalité et dans sa centralité, représentation propre à tout espace 
habité. C'est autant le « rêve de l'escalier » qui mène de la cave - lieu du secret - au grenier - lieu du souvenir -, en passant par la chambre et le séjour - lieux de vie -, que le « rêve de hutte » qui protège le moi qui sont convoqués pour penser l'école comme un espace habitable.

Par ailleurs ces étages tant désirés, parce qu'ils « font moins pauvres » mais aussi parce qu'ils seront distributeurs d'espace, devront être desservis par des escaliers et des ascenseurs menant dans des couloirs, avec fenêtres, donnant accès à de vastes salles aux usages bien spécifiés : une pour la musique, une autre pour le dessin, etc.

Enfin ce qu'ils redoutent par-dessus tout, c'est que ce qui leur est aujourd' hui presque impossible, à savoir : rester « tranquilles » dans les couloirs pour « parler ensemble », « se raconter », « discuter », « blaguer », « jouer à se bagarrer un peu », leur soit finalement interdit. Ils ajoutent que « ce qu'ils [les adultes] appellent les couloirs, sont en réalité des allées, des rues », dans lesquelles « on est dehors ». Ils expriment clairement le fait de n'être pas suffisamment protégés, contenus, par ce collège sans murs, aux quatre vents. Il n'y a pas d'entre-deux où séjourner parce qu' « on est direct dehors! », pas de murs qui protègent parce que ceux des préfabriqués sont « en carton » et que « le préau c'est un toit en tôle et quatre poteaux »... Ce qui devient alors prégnant, face aux plans du collège à construire, c'est la crainte d'être privé du collège, une fois celui-ci devenu plus protecteur et chaleureux, comme ils imaginent pouvoir être celui à venir et comme ils décrivent les autres collèges qu'ils ont fréquentés. C'est leur angoisse de n'y point trouver de place qui se fait jour : soit parce qu'ils seront déjà loin, soit parce que d'autres la leur prendront, soit encore parce que les adultes le leur interdiront. Aussi affirment-ils : «C'est sûr il y aura plus d'autorité », «On n'aura pas le droit de rester dans les couloirs, ils nous feront descendre », comme rétrogradés dans un rez-de-chaussée mal contenant auquel ils seraient comme destinés. C'est évidemment la symbolique de l'ascension, développementale, psychique ou sociale, qui apparaît aux nouages de ces escaliers manquants et désirés et de la crainte d'en être à jamais privés; comme si le préfabriqué symbolisait la condition même de ces jeunes, prédéfinis par une institution, c'est-à-dire par une société qui les promet à un avenir certain plutôt que de leur promettre un certain avenir.

Cette configuration spatiale ne semble pas totalement anéantir les relations entre pairs qui disent malgré tout se retrouver « là où il y a de l'herbe », « entre les deux blocs, là où il y a un banc », « en permanence » pour «se raconter», «pour faire des petits groupes ». En revanche, les relations avec la génération adulte semblent en pâtir. Les adultes de l'établissement ne s'attardent guère dans les allées et leur préfèrent la « salle des profs » interdite aux élèves qui la décrivent d'ailleurs comme un lieu 


\section{Dossier}

Un espace potentiel au collège

à part. Ils y perçoivent alors une forme d'injustice : «Ils ont chaud là-bas, y a des canapés et du café. Même des gâteaux! », " alors que nous on gèle dehors ». C'est tout le processus identificatoire qui en subit les conséquences : les préfabriqués deviennent des supports identificatoires négatifs. Cet état de fait, on le comprend, est inacceptable pour ces jeunes. Ainsi, alors que nous étions des « intervenants extérieurs », ils nous adressent ces mots très forts : «Regardez autour de vous, il n'y a que du gris, du pâle, c'est mort ici ! ». Et moi de leur répondre: « Je regarde, et ce que je vois c'est vous, et vous n'êtes ni pâles ni morts mais bien vivants et pleins de couleurs! »Alors ils s'écrient : «Ah oui madame, y a que nous qui sommes bien ici!»

Ce dernier point de dialogue me conduit à penser que ces quelques séances d'atelier ont fourni un étai, certes provisoire, à un processus identificatoire positif. En effet, ces jeunes gens ont su se saisir de ce que nous leur avons proposé : un temps de parole. Ils se sont exprimés librement sur le mode de la «variation autour d'un même thème ». Notre

L'informel est en effet, dans l'antre des institutions, le seul espace-temps où l'attention peut être flottante, où le corps peut se relâcher, où il est permis de jouer et de vagabonder, où il est possible de penser pour soi-même et non sur demande. souci premier fut d'être suffisamment à l'écoute de leurs dires et de leurs maux, pour que cette rencontre puisse être qualifiée, dans l'« après-coup », de transitionnelle, c'est-àdire qu'elle ait été intersubjective au sens plein.

La transitionnalité, au sens de Donald Winnicott (1971), fait défaut auprès de ces jeunes; ce « moment atelier », enclave d'un « moment adolescent » à l'intérieur du « moment école», fut une tentative de remédier à cet état de fait marqué par la précarité architecturale et par les conséquences de celle-ci sur les liens qui peinent à se tisser entre les habitants de ces lieux. Lieux qui pour devenir des espaces doivent être "pratiqués », ainsi que le postule Michel de Certeau (1980), et qui sont effectivement habités par les adolescents alors que les adultes ne font qu'y passer. C'est en cela peut-être qu'ils seraient pour certains des non-lieux, au sens de Marc Augé (1992), alors qu'ils sont des lieux pour d'autres. C'est d'ailleurs à cette condition seulement qu'ils deviennent habitables par les adolescents, c'est-à-dire praticables par eux selon leur propre mode d'habiter. Ce serait encore la capacité d'être seul en présence de l'autre, l'adulte mais aussi l'autre sexe ou encore le groupe, qui selon Winnicott (1958) serait une expérience paradoxale fondamentale à la constitution de l'identité subjective, qui trouverait à s'y construire. L'informel est en effet, dans l'antre des institutions, le seul espace-temps où l'attention peut être flottante, où le corps peut se relâcher, où il est permis de jouer et de vagabonder, où il est possible de penser pour soi-même et non sur demande. 


\section{DE L'INFORMEL AU TRANSITIONNEL}

Dans les établissements scolaires, le cadre, qu'avec Erving Goffman (1971) nous dirions « primaire », comprendrait notamment le programme, les horaires, le règlement intérieur. Ce serait le cadre formel mais il existe un cadre informel, peut-être plus variable mais relativement stable malgré tout, et qui connaît la particularité d'être l'œuvre des jeunes euxmêmes. C'est d'ailleurs ce que met en exergue l'étude de Julie Delalande (2001) sur la cour de récréation. Les sujets y inventent leurs propres modes de vie et mettent en pratique ce que Cornelius Castoriadis (1975) appelle « l'auto-institution de la société » qui est un principe fondamental de la démocratie. Or, il semble essentiel aujourd'hui de ne pas céder (sur) ce qui reste d'espaces de liberté et de création aux jeunes générations, et pour cela il faut pouvoir décrire et comprendre l'importance qu'ils revêtent.

L'informel, ce «bain environnemental » (Poizat, 2003) dans lequel nous sommes immergés, c'est ce bouillon qui répond assez bien à l'idée de culture : l'informel pourrait être ce lieu dans lequel on se transmet, à son su et à son insu, des représentations, des pratiques, auxquelles on confère du sens. C'est aussi le lieu de l'oralité et donc d'une tradition culturelle qui se transmet entre pairs et de génération en génération. Ce peut être aussi le lieu des rites, de leur invention, de leur passation et de leur transmission. L'informel serait encore, en regard des concepts d' « inachevé » soutenu par Georges Lapassade (1972) et d'« imaginaire » tel que Castoriadis (1975) l'articule à un devenir autonome, ce qui est en création, ce qui est potentiel, ce qui va advenir de/par/pour soi. C'est là que se construisent les objets culturels de demain : le langage, les écritures, les arts du corps et les arts plastiques. C'est là que les jeunes générations créent, trouvent et expérimentent les ressources qu'elles incarneront bientôt, parce que «c'est dans les espaces d'un relatif abandon par les pouvoirs publics que les jeunes construisent leurs inscriptions sociales » (Vulbeau, 2002, p. 174).

Aussi, plutôt que de définir l'informel uniquement par opposition au formel et au non-formel (Poizat, 2003 ; Brougère, Bézille, 2005), je souhaiterais mettre l'accent sur la dimension plus subjective de ce que l'on peut entendre sous ce vocable. Dans les institutions éducatives et thérapeutiques, les espaces et les temps formels peuvent être appréhendés comme des dispositifs. Ils sont agencés par des règles, des normes, des fonctions, ils sont délimités par des dénominations, des attributions d'horaires, de lieux, etc., ils sont le plus souvent stables et fiables, observables, inscrits dans une temporalité et une durée. Ces dispositifs se veulent favorables aux processus d'apprentissage et de socialisation en encadrant 
Un espace potentiel au collège

l'expérimentation que peuvent en faire les adolescents. Ce que je propose de définir comme informel serait, au contraire, ce qui n'est pas normé, organisé, pensé, utilisé en tant que tel par les institutions. L'informel serait alors ce que les individus investissent, en conférant ainsi des rôles, des fonctions et des enjeux singuliers et sociaux aux espaces et aux temps laissés vacants. L'informel peut être pensé comme le réceptacle possible de ce que ne peut comprendre le formel : les sensations, leur expression, les corps en mouvement, l'espace-temps de la rencontre avec soi-même et avec un autre, dans sa ressemblance et dans sa différence, le jeu et la créativité... Il s'agit là d'une dialectique à trois termes : l'informel en se formalisant devient le formel qui donne naissance à un nouvel informel.

Les espaces et les temps informels, en particulier ceux qui s'immiscent au cœur des institutions très formalisées telle que l'Éducation nationale, sont les lieux de l'entre-deux et du passage mais aussi les lieux de l'informe au sens de «ce qui attend de prendre forme ». Ils sont donc propices à l'acte de création, c'est-à-dire au jeu. Nous pourrions dire que les espaces et les temps informels seraient la matérialisation d'un « espace potentiel », une « aire infinie de séparation » qui seule permet qu'un lien véritable, un lien à l'autre en tant qu'autre, puisse se tisser. Pour Winnicott (1971), comme pour nombre de penseurs avant et après lui, l'être humain existe dans la relation à un autre. Cet autre est évidemment d'abord le parent, la mère, mais ne tarde pas à devenir le père et les pairs. C'est de la relation à l'environnement qu'il s'agit lorsque nous parlons d'intersubjectivité. Nous ne nous référerons pas ici au seul sujet mais bien au sujet parmi les sujets, pris dans le maillage relationnel: c'est parce qu'il y a un «tu » que le « je » peut advenir. Le transitionnel c'est le jeu, c'est-à-dire qu' « en jouant l'enfant manipule les phénomènes extérieurs, il les met au service du rêve et il investit les phénomènes extérieurs choisis en leur conférant la signification et le sentiment du rêve » (Winnicott, 1971, p. 105). Winnicott ajoute qu'il y a un lien direct entre les phénomènes transitionnels, le jeu, le jeu partagé et les expériences culturelles, tout comme il y a un lien entre les espaces privés, publics et communs. Ces liens entre les espaces institutionnels formalisés, qu'ils soient privés comme la famille, ou publics comme la rue et l'école, sont articulés par des espaces communs. Le concept d'interstices (Roussillon, 1987), ces seuils qui font lien en même temps et parce qu'ils séparent, nous permet de mieux comprendre l'informel comme un « espace potentiel ». L'interstice est ce qui est entre, entre-deux, intervalle, dans lequel trouvent à s'immiscer, à circuler, parfois à se déposer, les objets et les sujets. Selon René Roussillon l'informe est lui aussi en quête de lieux où se déposer. 
Les interstices, lorsqu'ils sont repérés, font l'objet d'une certaine tolérance par les institutions. La pléthorique littérature en sociologie des organisations a largement couvert ce point (Crozier, Friedberg, 1977) qui ne fut pas non plus en reste chez les institutionnalistes à commencer par Michel Foucault (1975).

Les exigences du formel, de «l'école assise » décriée par Adolphe Ferrière (1922), font certes enveloppe contenante (Bion, 1962 ; Anzieu, 1985) dans une certaine mesure, mais la frontière est mince entre la contenance et la contention. Les interstices fournissent la possibilité de reprendre son souffle dans un holding (Winnicott, 1960) plus adéquat puisque à la fois intérieur à l'établissement mais suffisamment souple pour y circuler librement. Les interstices, et les seuils qu'ils comprennent, sont et font passages entre les espaces, entre les

Nous pourrions dire que les espaces et les temps informels seraient la matérialisation d'un « espace potentiel », une « aire infinie de séparation » qui seule permet qu'un lien véritable, un lien à l'autre en tant qu'autre, puisse se tisser. temps, c'est-à-dire qu'ils permettent une transition entre les postures, les rôles et les fonctions de chacun. On peut dire qu'ils sont chargés symboliquement, imaginairement et matériellement. C'est une « aire intermédiaire d'expérience », dirait Winnicott (1971), dans laquelle, ainsi qu'avant lui le disait Georg Simmel, l'expérience serait le lieu d'indétermination entre le sujet et l'objet.

\section{POUR CONCLURE PROVISOIREMENT}

Les politiques éducatives actuelles, redoutant sans doute le changement que ne manque pas de faire advenir le processus d'un « moment adolescent » pris dans le «moment école », semblent s'accorder pour exercer un contrôle serré de ces espaces et de ces temps quand elles ne vont pas jusqu'à les supprimer tout bonnement. Je postulerai au contraire qu'il s'agit de faire, ou de laisser, une place à cet autre-cadre, capable d'accueillir les corps et de voir naître une culture adolescente, afin que les cadres pédagogiques puissent recouvrir d'autres fonctions, non moins indispensables, à la manière d'une dialectique ou mieux, d'un dialogue. Cette vacance - laissée à et constituée par l'informel institutionnel - n'est donc pas vacuité puisque les adolescents, ceux que j'ai observés, mais aussi celle que j'ai été et ceux que je côtoyais alors, savent s'emparer de ces lieux et de ces moments pour se rencontrer, se retrouver et se construire dans un déjà-là institué toujours à transformer. Aussi faut-il tenter de suivre le programme suggéré par Alain Vulbeau et «s'intéresser à la question du normatif sous le double aspect de l'invention normative et de la socialisation, plus ou moins problématique, de cette invention ; 
Un espace potentiel au collège

puis réfléchir aux pratiques des jeunes, non comme des pratiques "indigènes" et/ou "sauvageonnes", mais comme des processus de socialisation secondaires et de rencontres potentielles avec les institutions » (Vulbeau, 2001, p. 14). Ce serait là la gageure d'une transmission transitionnalisée plutôt que ritualisée, mais toujours ancrée dans un espacetemps social partageable et partagé.

\section{BIBLIOGRAPHIE}

Anzieu D., Le moi-peau, Dunod, coll. « Psychismes », Paris, 1995 (1 1 éd. 1985).

Anzieu D., Les enveloppes psychiques, Dunod, coll. «Inconscient et culture », Paris, 2003 (1re éd. 1987).

Augé M., Non-lieux. Introduction à une anthropologie de la surmodernité, Le Seuil, coll. « La librairie du Xx siècle », Paris, 1992.

Bachelard G., La poétique de l'espace, Presses universitaires de France, coll. «Quadrige. Grands textes », Paris, 1981 (1re éd. 1957).

Bıon W., Aux sources de l'expérience, Presses universitaires de France,

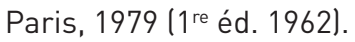

Castoriadis C., L'institution imaginaire de la société, Le Seuil, coll.

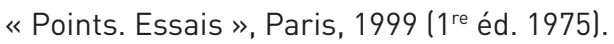

Certeau M. (DE), L'invention du quotidien. Vol. I, « Arts de faire», Gallimard, coll. « Folio essais », Paris, 1990 (1 re éd. 1980).

Chabert C., Psychanalyse et méthodes projectives, Dunod, coll. « Les topos. Psychologie », Paris, 1998. 
Crozier M., Friedberg E., L'acteur et le système. Les contraintes de l'ac-

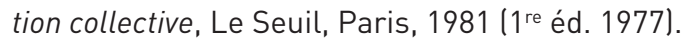

Delalande J., La cour de récréation. Contribution à une anthropologie de l'enfance, Presses universitaires de Rennes, coll. « Le sens social », Rennes, 2001.

Dufour D.-R., L'art de réduire les têtes. Sur la nouvelle servitude de l'homme libéré, à l'ère du capitalisme total, Denoël, coll. «Grand format médiations », Paris, 2003.

Ferrière A., L'école active, Neuchâtel, Éditions Forum, Genève (Suisse), 1922.

Foucault M., Surveiller et punir. Naissance de la prison, Gallimard, coll. «Tel », Paris, 1993 (1 1 re éd. 1975).

GAVARINI L., «Des groupes de parole avec les adolescents. À la recherche d'une parole "autre" », Cliopsy, n 1, 2009, pp. 51-68 (téléchargeable sur www.revue.cliopsy.fr).

Goffman E., La mise en scène de la vie quotidienne, Minuit, coll. « Le

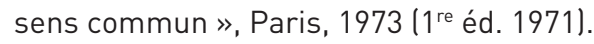

LAPASSADE G., L'entrée dans la vie. Essai sur l'inachèvement de l'homme, Paris, Union générale d'éditions, 1972.

MAsson A., «Le "moment adolescent" entre saisie par l'instant et constitution du présent », Figures de la psychanalyse, « L'adolescent vers la psychanalyse », vol. IX, 2004, pp. 103-126.

MaURIN A., «Passages adolescents : leurs matérialisations dans les espaces et les temps informels des institutions éducatives », Conserveries mémorielles, $\mathrm{n}^{\circ}$ 7, 2010 (en ligne sur http://cm.revues.org).

MAURIN A., «Les passages adolescents : habiter les interstices », Le Télémaque, n³8, 2010 (à paraître).

Poizat D., L'éducation non formelle, L'Harmattan, coll. «Éducation comparée », Paris, 2003.

RoussiLlon R., « Espaces et pratiques institutionnelles. Le débarras et l'interstice », in KAES R., et al., L'institution et les institutions. Études psychanalytiques, Dunod, coll. « Inconscient et culture », Paris, 2003

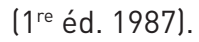

VACHeret C., Pratiquer les médiations en groupes thérapeutiques, Dunod, coll. «Psychothérapies », Paris, 2002.

Vulbeau A., La jeunesse comme ressource. Expérimentations et expérience dans l'espace public, OBVIES/Éres, coll. «Questions vives sur la banlieue », Toulouse, 2001. 
Un espace potentiel au collège

Vulbeau A., Les inscriptions de la jeunesse, L'Harmattan, coll. « Débats Jeunesses », Paris, 2002.

Winnicott D. W., Jeu et réalité. L'espace potentiel, Gallimard, coll. « Folio essais », Paris, 2002 (1re éd. 1971).

Winnicott D. W., «La capacité d'être seul »; « La théorie de la relation parents-nourrissons », De la pédiatrie à la psychanalyse, Payot, coll. «Science de l'homme », Paris, 2007 (1'res éd. 1958 et 1960).

\section{L'AUTEURE}

\section{Aurélie Maurin}

aurelie.maurinayahoo.fr

Psychologue clinicienne et psychothérapeute d'enfants et d'adolescents, doctorante, enseigne les sciences de l'éducation à l'université Paris-VIII en qualité de monitrice.

Thèmes de recherche : les espaces et les temps informels dans les institutions éducatives; les constructions identitaires et le processus adolescent.

\section{A notamment publié}

MAURIN A., « Bruit-silence, d'une posture à l'autre, le contre-transfert d'une retranscriptrice », Cliopsy, n², 2009, pp. 49-57 (téléchargeable sur www.revue.cliopsy.fr).

MAURIN A., «Passages adolescents : leurs matérialisations dans les espaces et les temps informels des institutions éducatives », Conserveries mémorielles, n 7, 2010 len ligne sur http://cm.revues.org).

MAURIN A., «Les passages adolescents : habiter les interstices », Le Télémaque, n 38 , 2010 là paraître). 